\title{
Cissampelos (Menispermaceae) in the state of Pará, Brazil
}

\author{
Julio dos Santos de Sousa ${ }^{1, *}$ (i) \& Ely Simone Cajueiro Gurgel ${ }^{2}$ (i) \\ 'Museu Paraense Emílio Goeldi, Programa de Capacitação Institucional (PCI), Campus de Pesquisa, \\ Avenida Perimetral, 1901, Terra Firme, CEP 66077-830, Belém, Pará, Brasil. \\ "Author for correspondence: jssousa27@yahoo.com.br \\ ${ }^{2}$ Museu Paraense Emílio Goeldi, Coordenação de Botânica-COBOT, Avenida Perimetral, \\ 1901, Terra Firme, CEP 66077-830, Belém, Pará, Brasil.
}

Recebido em 24.I.2020

Aceito em 10.1X.2021

DOI 10.21826/2446-82312022v77e2022004

ABSTRACT - The study presents the taxonomic treatment of Cissampelos L. for the state of Pará. Botanical specimens from national and foreign herbaria, as well as 14 new collections and the literature, were analyzed. The genus is represented by seven species: Cissampelos ovalifolia DC., C. andromorpha DC., C. laxiflora Moldenke, C. pareira L., C. fasciculata Benth., C. glaberrima A.St.-Hil. and C. tropaeolifolia DC. The last three species are new records for the state of Pará. An identification key, descriptions, illustrations, distribution data and additional comments for each species are provided.

Keywords: Amazonia, Capena, Cissampelideae, Menispermoideae, taxonomy

RESUMO - Cissampelos L. (Menispermaceae) no estado do Pará, Brasil. O estudo apresenta o tratamento taxonômico de Cissampelos L. para o estado do Pará. A metodologia abrangeu a análise do material proveniente de 14 expedições de coletas e exsicatas de herbários nacionais e estrangeiros, além de literatura especializada. O gênero está representado na área por sete espécies: Cissampelos ovalifolia DC., C. andromorpha DC., C. laxiflora Moldenke, C. pareira L., C. fasciculata Benth., C. glaberrima A.St.-Hil. e C. tropaeolifolia DC. As três últimas espécies são novas ocorrências para o estado do Pará. São apresentadas chave de identificação, descrições, ilustrações, bem como dados adicionais sobre distribuição geográfica e comentários das espécies.

Palavras-chave: Amazônia, Capena, Cissampelideae, Menispermoideae, taxonomia

\section{INTRODUCTION}

Menispermaceae (the moonseed family) comprises two subfamilies (Menispermoideae Arn. and Chasmantheroideae Luerss.), nine tribes, 72 genera and approximately 526 species distributed mainly in tropical regions of the world (Ortiz et al. 2016). In Brazil, the family is represented by 15 genera, 108 species (29 endemic), six subspecies and seven varieties (BFG 2015).

The tribe Cissampelideae Hook. f. \& Thomson comprises five genera and 128 species distributed in South and Central America, North America, Indomalesia, eastern Asia, Africa, and Madagascar (Ortiz et al. 2016). These authors place the genus Cissampelos L. within this tribe and subfamily Menispermoideae Arn.

Cissampelos is the only genus of the tribe Cissampelideae in Brazil, comprises 23 species (Ortiz et al. 2016) and has a pantropical distribution (Ortiz \& Nee 2014, Peña-Chocarro \& Egea 2015). In Brazil, the genus is represented by nine species (one endemic) distributed in the Amazonia, Caatinga, Cerrado, Atlantic Forest, Pampa and Pantanal (BFG 2015) phytogeographic domains and occurs in all states (SpeciesLink 2019).

The last review of Cissampelos was made by Rhodes (1975), who recognized 20 species, including nine in the Americas, nine in Africa, one in Asia and the pantropical Cissampelos pareira $\mathrm{L}$.

In Brazil, studies involving Cissampelos include taxonomic treatments of local floras or restricted protected areas that generally have few species, such as Costa \& Sano (2007) in São Paulo (Southeast), Teixeira \& Amorim (2012) in Bahia (Northeast), Sothers et al. (1999) in Ducke reserve (Amazonas) and Lima \& Teixeira (2018) for the canga of Serra of the Carajás (Pará), in the North Region.

Within this context, the present study was carried out to better understand the morphology and taxonomy of the species of Cissampelos in Pará and to provide reliable characters for identification. 


\section{MATERIAL AND METHODS}

The study was conducted using new collections from 14 expeditions and botanical specimens from national and foreign herbaria, principally in northern Brazil: EAFM, HAMAB, IAN, INPA, MG, MIRR, RON and UFACPZ (abbreviations according to Thiers, continuously updated), as well as the collections of MFS, NY, R, RB, SP, UB and collections nomenclatural types of Cissampelos. Morphological analyses and laboratory measurements were performed with the aid of a stereomicroscope. The illustrations were made by the main author and Carlos Alvarez. The species descriptions are based on the material studied. We analyzed material from the study areas and, when necessary, additional collections were consulted. The terminology of vegetative and reproductive parts follows Barroso et al. (1999), Gonçalvez \& Lorenzi (2011), Hickey (1973), Radford et al. (1974), Rizzini (1977), and Weberling (1992). Species identifications are based on analyses of types, keys and literature. The following abbreviations are used: fl (flower), fr (fruit) and sn (without a number).

\section{RESULTS AND DISCUSSION}

\section{Cissampelos L., Sp. Pl. 2: 1031. 1753.}

Liana or xylopodial herb. Leaves peltate or basifixed. Inflorescences axillary, terminal or cauliflorous, thyrsiform, racemose or paniculiform. Flowers actinomorphic, dichlamydeous. Staminate flowers with 4-5, valvular, membranous sepals; corolla ciatiform, cupuliform or patelliform, membranous. Pistillate flowers with 1 sepal and 1 petal. Stamens connate, forming a glabrous synandrium; anthers 4-6, extrorse, transversely dehiscent. Carpel 1, stigma trifid. Drupes subglobose to obovoid; epicarp membranous; mesocarp mucilaginous; endocarp hypocrepiform, condyle with lateral intrusion; seed curved, endosperm smooth.

Type species: Cissampelos pareira L., Sp. Pl. 2:1031. 1753.

\section{Identification key to the species of Cissampelos L. in Pará}

1. Leaf blade basifixed, venation basal actinodromous.

2. Xylopodial herb, 27-90 cm tall; petiole velutinous; leaf blade subcoriaceous, margin velutinous

\section{Cissampelos ovalifolia}

2. Liana, 1.1-12 m tall; petiole strigose, puberulent, tomentose or glabrous; leaf blade membranous to chartaceous, margin strigose or pubescent.

3. Petiole canaliculate; staminate flowers with 4 anthers; pistillate flowers with ellipsoid, obovoid or pyriform, velutinous ovary.

4. Abaxial surface of leaf blade sparsely strigose, apex acute, obtuse or rounded, margin rectilinear; staminate and pistillate inflorescences cauliflorous and axillary; staminate flower corolla cupuliform, glabrous, synandrium 0.5-0.9 mm long; pistillate flower petal oval or obtrullate, glabrous, ovary ellipsoid or pyriform

Cissampelos andromorpha

4. Abaxial surface of leaf blade pubescent or tomentose, apex acuminate or emarginate, margin repand; staminate inflorescences axillary; pistillate inflorescences axillary and terminal; staminate flower corolla patelliform, sericeous, synandrium sessile; pistillate flower petal deltoid, obovate or suborbicular, sericeous, ovary obovoid

Cissampelos fasciculata

3. Petiole not canaliculate; staminate flowers with 4-6 anthers; pistillate flowers with gibbous-turbinate or globose, pubescent or tomentose ovary.

5. Petiole glabrous or sparsely strigose; pulvinus twisted only in the proximal region; leaf blade glabrous on both surfaces, primary veins 5 ; staminate inflorescences thyrsiform; staminate bracts lanceolate or elliptic; staminate flowers cream, corolla glabrous; pistillate flower sepal obovate, dorsally pubescent, petal deltoid or suborbicular, glabrous, ovary gibbous-turbinate, pubescent, stigma deltoid Cissampelos laxiflora

5. Petiole tomentose; pulvinus erect in the distal and proximal regions; leaf blade strigose or sericeous on adaxial surface, sericeous or tomentose on abaxial surface, primary veins 5-9; staminate inflorescences paniculiform; staminate bracts deltoid or subcordiform; staminate flowers greenish yellow, corolla puberulent; pistillate flower sepal flabelliform or trullate, sericeous, petal flabelliform or reniform, sericeous, ovary globose, tomentose, stigma falciform

Cissampelos pareira

1. Leaf blade peltate, venation suprabasal actinodromous. 
6. Petiole glabrous or sparsely strigose; leaf blade glabrous on both surfaces, margin rectilinear or repand, glabrous; staminate bracts oval or deltoid; pistillate bracts glabrous or tomentose; staminate flowers greenish, synandrium 0.1-0.8 mm long; pistillate flower sepal elliptic, obovate or trullate, glabrous or sparsely puberulent, ovary glabrous, stigma deltoid; drupes glabrous Cissampelos glaberrima

6. Petiole sericeous or hirsute; adaxial surface sericeous or pubescent, abaxial surface sericeous, margin crenulate, sericeous; staminate bracts elliptic; pistillate bracts puberulent only on the margins; staminate flowers white or cream, synandrium 0.1-0.6 mm long; pistillate flower sepal cucullate or suborbicular, sericeous, ovary sparsely strigose, stigma narrow-triangular; drupes sparsely sericeous Cissampelos tropaeolifolia

Cissampelos andromorpha DC., Syst. Nat. 1: 539. 1817. (Fig. 1 A-C)

Liana, 1.5-6.5 m tall; stem voluble, glabrous or rarely sericeous; rhytidome brown or yellowish, grooved. Petiole $2.5-8.4 \times 0.4-1.5 \mathrm{~mm}$, subcylindrical, canaliculate, basifixed, sparsely strigose; pulvinus twisted only in the proximal region. Leaf blade $3.2-9 \times 3-9.3 \mathrm{~cm}$, basifixed, cordiform, membranous to chartaceous, flat, adaxial surface glabrous or sparsely strigose, abaxial surface sparsely strigose; apex acute, obtuse or rounded; base bilobed, subcordate; margin rectilinear, not revolute, strigose. Venation basal actinodromous, primary veins 5-7, palmatinerved; secondary veins diverging from the lower third of the blade; tertiary veins scalariform. Staminate inflorescences paniculiform, cauliflorous and axillary. Pistillate inflorescences in simple racemes, cauliflorous and axillary. Staminate bracts $0.3-1.3 \times 0.3-0.9 \mathrm{~cm}$, lanceolate or oval, sericeous to tomentose, or ebracteate. Pistillate bracts 1.1-9 × 0.3-9 cm, oval, elliptic or suborbicular, sericeous. Staminate flowers with white or yellowish perianth; sepals $4,0.5-1.9 \times 0.25-1.2 \mathrm{~mm}$, elliptic, oblanceolate or obovate, sericeous; corolla $0.4-1 \times 0.5-1.3$ $\mathrm{mm}$, cupuliform, glabrous; stamens connate, forming a claviform synandrium (0.5-0.9 mm long), anthers 4 . Pistillate flowers with greenish perianth; sepal 0.9-1.7 $\times$ $0.3-1.1 \mathrm{~mm}$, elliptic, lanceolate or spatulate, sericeous; petal $0.2-1.1 \times 0.3-1.2 \mathrm{~mm}$, oval or obtrullate, glabrous; ovary $0.5-1 \times 0.4-0.7 \mathrm{~mm}$, ellipsoid or pyriform, velutinous; stigma deltoid. Drupes $0.3-1 \times 0.4-0.9 \mathrm{~cm}$, obovoid or subglobose, yellow or red, sparsely puberulent; endocarp with lateral and dorsoventral, spiciform projections.

Examined material: BRAZIL, PARÁ, Altamira, Belo Monte, Rio Xingu, X-XII.2007, fl., R. P. Salomão 1030 (MG); Altamira, Belo Monte, Rio Xingu, X-XII.2007, fr., R. P. Salomão 1031 (MG); Altamira, Juruá, 27.XI.1986, fl. \& fr., S. A. M. Souza et al. 561 (MG); Rio Xingu basin, 24.XI.1980, fl., G. T. Prance et al. P26433 (MG); Belém, IAN, 17.XII.1944, fl., R. L. Fróes 20771 (NY); Belém, km 15 (APEG), 04.I.2000, fl., M. R. Cordeiro 4810 (IAN); Belém, Municipal Bosque, 16.X.1945, J. M. Pires \& G. A. Black 439 (IAN); Belém, 03.X.1945, J. M. Pires \& G. A. Black 346 (IAN); Belém, IAN, 06.XI.1942, W.A. Archer 777 (IAN); Belém, 5 km from IAN, 29.IX.1942, fl., M. B.
Silva 119 (IAN); Belém, IAN, 05.X.1950, G. A. Black \& P. Ledoux 50-10225 (IAN); Belém, IAN, 08.III.1955, fr., T. N. Guedes 323 (IAN); Belém, IAN, 10.IV.1957, fl., G. A. Black 57-19252 (IAN); Belém, IPEAN, s.d., fr., J. M. Pires \& N. T. Silva 10464 (IAN); Belém, IPEAN, Mocambo reserve, 18.IX.1963, fl., E. Oliveira 2588 (IAN); Belém, IPEAN, XII.1965, fr., B. G. Schubert 2247 (IAN); Belém, IPEAN, 21.V.1975, R. R. Santos \& J. F. V. Carvalho 362 (IAN); Belém, Museu, 05.XII.1912, J. Huber 12459 (MG); Belém, Tererê lane, 25.IX.1945, fl., J.M. Pires \& G. A. Black 267 (IAN, INPA, NY); railroad Salgado, 26.XI.1907, fl., A. Ducke 8907 (MG); Barra road, 28.IX.1898, fl., M. Guedes 1604 (MG); Railroad Bragança, João Coelho, 14.III.1947, fr., J.M. Pires \& G. A. Black 1420 (MG); road on BR 163, Cuiabá-Santarém, km 890, 14.II.1977, fl., J. H. Krkbride \& E. Lleras 2768 (MG); IgarapéAçu, FCAP, capoeira from Urucu, 16.I.1996, fr., F .P. M. Oliveira et al. 40 (MG); Ilha do Marajó, Capoeira from Condeixa, 15.XI.1948, fl., G. A. Black 48-3578 (IAN); Melgaço, Caxiuanã, 04.IV.2004, fr., M. A. Freitas et al. 1084 (MG); s.l., 11.XI.1948, fl., N. T. Silva 164 (IAN); Monte Dourado, Rio Jarí, Serrinha, 02.XII.1968, fl., N. T. Silva 1478 (IAN); Oriximiná, 22.XII.1910, fr., A. Ducke 11487 (MG); Oriximiná, $1 \mathrm{~km}$ from the right bank of the Rio Mapuera, 15.VIII.1986, fl., F.C.A. Cid et al. 7765 (MG); Pau d'Arco, Marajoara, s.d., fl., J. Grogan 295 (MG; IAN); Rio Cumina-mirim, 16.XII.1906, fl., A. Ducke 7972 (MG); Cuiabá-Santarém highway, BR 163, km 1223, near Kazuo igarape, 21.XI.1977, fr., G. T. Prance et al. 25636 (MG); Tucuruí highway, right bank, km 41-45, 07.IV.1981, fr., N. A. Rosa et al. 4078 (MG); Santarém, road of the Lama igarapé, Poção, 19.VI.1954, fl., R. L. Fróes 30842 (IAN); Santarém, road of the Lama igarapé, Poção, 19.VI.1954, fr., R. L. Fróes 30867 (IAN); São Miguel do Guamá, bank of the Rio Irituia, 29.X.1948, fl. \& fr., Back \& Foster 48-3341 (IAN); Serra do Cachimbo, BR 163, Cuiabá-Santarém highway, km 845, 18.II.1977, fl. \& fr., J. H. Kirkbride \& E. Lleras 2881 (MG).

Distribution: Costa Rica, Guatemala, Nicaragua, Panama, Bolivia, Brazil, Colombia, Ecuador, Guyana, French Guiana, Suriname, Peru and Venezuela (MBG 2019, Rhodes 1975, Teixeira \& Amorim 2012). In Brazil, this species occurs in Acre, Amazonas, Pará, Rondônia, Roraima, Tocantins, Alagoas, Bahia, Ceará, Maranhão, Paraíba, Pernambuco, 


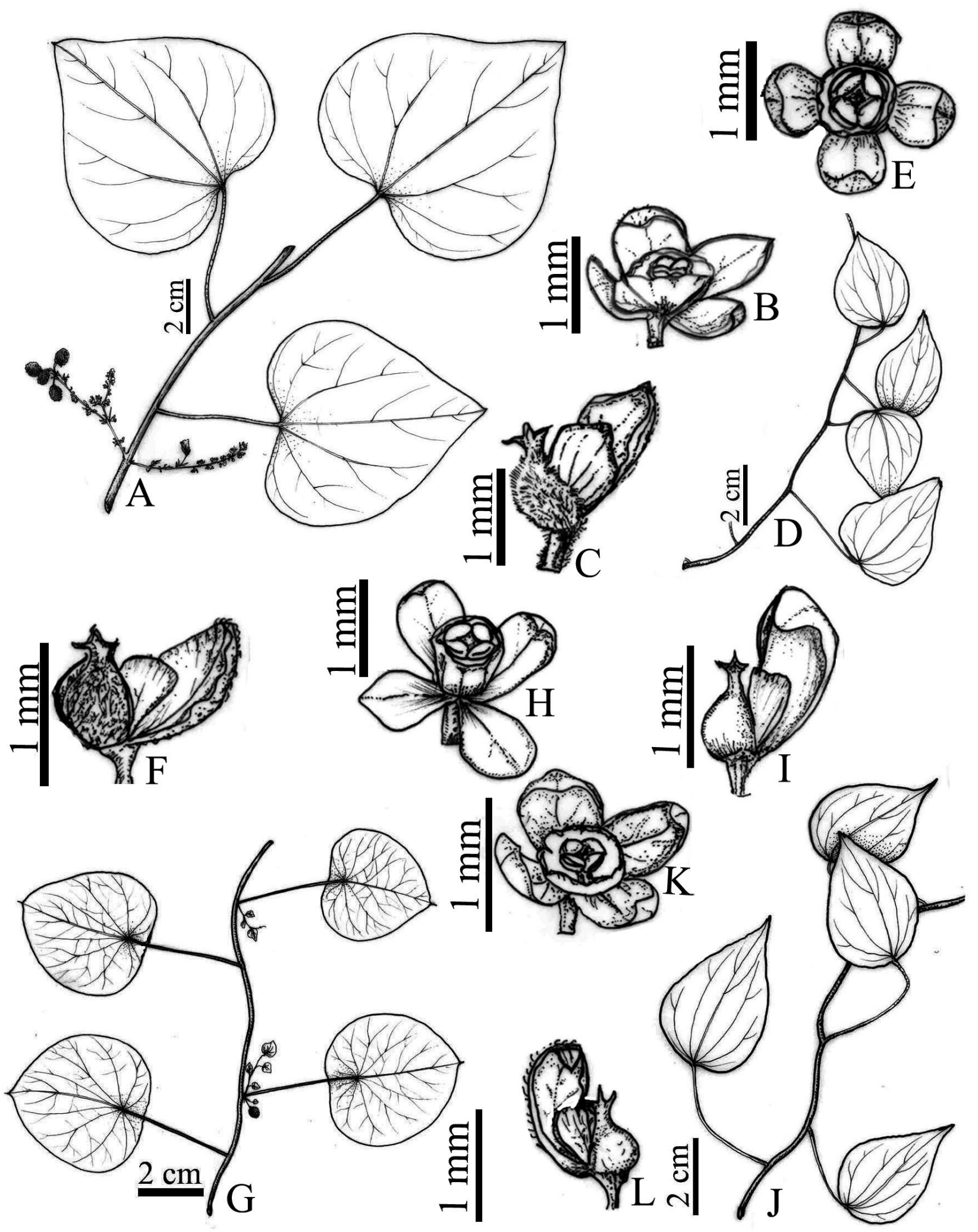

Figura 1. Species of Cissampelos in Pará state, Brazil. A-C. Cissampelos andromorpha. A. branch with pistillate flowers and drupes; B. staminate flower: C. pistillate flower. D-F. Cissampelos fasciculata; D. habit; E. staminate flower; F. pistillate flower. G-I. Cissampelos glaberrima; G. branch with drupe; H. staminate flower; I. pistillate flower. J-L. Cissampelos laxiflora; J. branch detail; K. staminate flower; L. pistillate flower. 
Sergipe, Goiás, Mato Grosso, Espírito Santo, Minas Gerais, Rio de Janeiro, São Paulo, Paraná, Rio Grande do Sul and Santa Catarina, in the Amazonia, Caatinga, Cerrado, Atlantic Forest, Pampa and Pantanal phytogeographic domains (BFG 2015, Lima \& Teixeira 2018).

Among the species studied, C. andromorpha is easily identified by the cauliflorous and axillary inflorescences on the same plant, ellipsoid or pyriform ovary and endocarp with lateral and dorsoventral, spiciform projections.

Cissampelos fasciculata Benth., London J. Bot. 2: 361 . 1843.

$$
\text { (Fig. } 1 \text { D-F) }
$$

Liana, 3-12 m tall.; stem voluble, puberulent; rhytidome brown or yellowish, not fissured. Petiole 3.5$10.4 \times 0.4-1.4 \mathrm{~mm}$, subcylindrical, canaliculate, basifixed, puberulent; pulvinus twisted only in the proximal region. Leaf blade 4.3-14 × 4.5-15.1 cm, basifixed, cordiform, membranous to chartaceous, flat, adaxial surface glabrous or pubescent, abaxial surface pubescent or tomentose; apex acuminate or emarginate; base bilobed, subcordate to cordate; margin repand, not revolute, strigose. Venation basal actinodromous, primary veins 5-9, palmatinerved; secondary veins diverging from the lower third of the blade; tertiary veins scalariform. Staminate inflorescences in racemes or paniculiform, axillary. Pistillate inflorescences in racemes or paniculiform, axillary and terminal. Staminate bracts $0.2-0.7 \times 0.15-0.6 \mathrm{~cm}$, cordate or oval, velutinous. Pistillate bracts $0.3-2 \times 0.3-1.9 \mathrm{~cm}$, cordate or subdeltoid, velutinous. Staminate flowers with greenish perianth; sepals $4,0.5-1.6 \times 0.4-1.1 \mathrm{~mm}$, elliptic or obovate, puberulent or velutinous; corolla $0.5-1.4 \times 0.4-1.4 \mathrm{~mm}$, patelliform, sericeous; stamens connate, forming a sessile, claviform synandrium, anthers 4 . Pistillate flowers with greenish perianth; sepal $0.9-1.8 \times 0.5-1 \mathrm{~mm}$, oblanceolate or obovate, puberulent or velutinous; petal $0.4-1.1 \times 0.3-1.2$ $\mathrm{mm}$, deltoid, obovate or suborbicular, sericeous; ovary 0.5-1.2 $\times 0.4-0.9 \mathrm{~mm}$, obovoid, velutinous; stigma deltoid. Drupes $0.4-0.7 \times 0.3-0.6 \mathrm{~cm}$, obovoid, orangish to red, puberulent; endocarp with dorsal and lateral, tuberculate projections.

Examined material: BRAZIL, PARÁ, approximately 70 $\mathrm{km}$ from Tucuruí, $65 \mathrm{~km}$ SSW on old BR 422, then $5 \mathrm{~km}$ NW on new logging road, 18-20.XI.1981, fl., D. C. Daly et al. 1433 (NY); Conceição do Araguaia, white sand island, 07.VIII.1978, fl., N. T. Silva 4761 (NY); Rio Tocantins, near igarape Cajazeirinha, approximately $30 \mathrm{~km} \mathrm{~N}$ of Itapiranga, 27.XI.1981, fr. \& fl., D. C. Daly 1586 (NY).

Additional examined material: BRAZIL, AMAZONAS, Barcelos, vicinity of Tototobi Mission, Yanomami Indian village, tributary of Rio Demeni, 23.VIII.1975, fl. \& fr., G. T. Prance \& J. F. Ramos 23631 (INPA, NY); falls of Madeira, X.1886, fl., H. H. Rusby 1442 (NY); Manaus, BR 174, 28.I.1955, fr., W. Rodrigues 716 (INPA); MINAS
GERAIS, Ituiutaba, Santa Terezinha, 23.I.1948, fl., A. Macedo 1178 (NY).

Distribution: Brazil, Colombia, Costa Rica, Ecuador, Guyana, Mexico, Nicaragua, Guatemala, Panama, Peru, Suriname and Venezuela (MBG 2019, Rhodes 1975). In Brazil, this species occurs in Amazonas and Minas Gerais, in the Amazonia and Cerrado phytogeographic domains (BFG 2015, Rhodes 1975). This species is here reported for the first time for the state of Pará.

Cissampelos fasciculata is easily distinguished from congeners by the acuminate or emarginate leaf apex and sessile synandrium. Collections from the region are notoriously confused with C. andromorpha; however, C. fasciculata is differentiated by its leaf blades with a pubescent or tomentose abaxial surface and acuminate or emarginate apex, axillary staminate inflorescences and sessile synandrium, whereas the second species has a sparsely strigose abaxial surface, acute, obtuse or rounded apex, cauliflorous and axillary staminate inflorescences and a synandrium $0.5-0.9 \mathrm{~mm}$ long.

Cissampelos glaberrima A.St.-Hil., Fl. Bras. Merid. 1:57. 1825.

(Fig. 1 G-I)

Liana, 2-3 m tall; stem voluble, glabrous; rhytidome brown, striate. Petiole $3.2-7 \times 0.5-1 \mathrm{~mm}$, subcylindrical, canaliculate, peltate (inserted $5-13 \mathrm{~mm}$ from the base), glabrous or sparsely strigose; pulvinus twisted only in the proximal region. Leaf blade $3.2-7.2 \times 3.4-7.3 \mathrm{~cm}$, peltate, oval or suborbicular, membranous to chartaceous, flat, glabrous on both surfaces; apex acute; base entire, rounded or truncate; margin rectilinear or repand, not revolute, glabrous. Venation suprabasal actinodromous, primary veins $9-12$, plinerved; secondary veins diverging from the lower third of the blade; tertiary veins reticulate. Staminate and pistillate inflorescences racemose, axillary. Staminate bracts $0.4-1.2 \times 0.4-1.5 \mathrm{~cm}$, oval or deltoid, sparsely sericeous. Pistillate bracts $0.3-1.3 \times 0.4-1.7 \mathrm{~cm}$, cordiform or reniform, glabrous or tomentose. Staminate flowers with greenish perianth; sepals 4, 0.9-1.7 × 0.7-1 $\mathrm{mm}$, elliptic or obovate, glabrous; corolla $0.6-0.9 \times 0.5-1$ $\mathrm{mm}$, cupuliform, glabrous; stamens connate, forming a claviform synandrium $(0.1-0.8 \mathrm{~mm}$ long), anthers 4 . Pistillate flowers with greenish to yellowish perianth; sepal 1.2-1.8 $\times 0.8-1 \mathrm{~mm}$, elliptic, obovate or trullate, glabrous or sparsely puberulent; petal $0.5-1 \times 0.5-1 \mathrm{~mm}$, flabelliform or suborbicular, glabrous; ovary $0.5-1 \times$ 0.4-0.6 mm, gibbous, glabrous; stigma deltoid. Drupes 0.3-0.7 × 0.3-0.6 cm, obovoid, red, glabrous; endocarp with lateral and dorsoventral, tuberculate projections.

Examined material: BRAZIL, PARÁ, Acará, 06.I.2017, fl., J. S. Sousa 722 (MG); Viseu, Jutaí, Serra do Piriá, terra firme forest, 28.V.2010, fr., R. C. Forzza 5884 (MG, RB). 
Additional examined material: BRAZIL, MINAS GERAIS, Belo Horizonte, 24.IX.1942, fl., M. Magalhães 2195 (IAN); Carandaí, Crespo, 18.XI.1946, fl., A. P. Duarte 553 (NY); Road from Viçosa to São Miguel, km 20, 23.XII.1929, fl. \& fr., Y. E. J. Mexia 4152 (NY); Viçosa, ESA - Viçosa, 29.III.1935, fl., J. G. Kuhlmann 2117 (NY).

Distribution: Bolivia, Brazil, Colombia, Costa Rica, Ecuador, Guatemala, Paraguay, Peru and Venezuela (MBG 2019, Rhodes 1975). In Brazil, this species occurs in Acre, Alagoas, Bahia, Ceará, Maranhão, Pernambuco, the Distrito Federal, Goiás, Mato Grosso, Minas Gerais, Rio de Janeiro and São Paulo, in the Amazonia, Caatinga, Cerrado, Atlantic Forest and Pantanal phytogeographic domains (BFG 2015). The species is here reported for the first time for the state of Pará.

Cissampelos glaberrima is the only congeneric species with glabrous leaves, ovary and drupes. According to Costa \& Sano (2007), this species has staminate flowers with spatulate sepals However, in the analyzed samples, the staminate flowers had elliptic or obovate sepals, which corroborates an analysis made by Rhodes (1975).

Cissampelos laxiflora Moldenke, Phytologia 2:215. 1947. (Fig. 1 J-L.)

Liana, 1.1-2.4 m tall; stem voluble, glabrous; rhytidome brown or greyish, fissured. Petiole 2.3-7.6 $\times 0.5-1 \mathrm{~mm}$, cylindrical, not canaliculate, basifixed, glabrous or sparsely strigose; pulvinus twisted only in the proximal region. Leaf blade $2.5-6.9 \times 1.8-4.6 \mathrm{~cm}$, basifixed, subcordiform or oval, membranous to chartaceous, flat, glabrous on both surfaces; apex acute, attenuate or mucronate; base entire or bilobed, rounded, subcordate to truncate; margin rectilinear, not revolute, strigose. Venation basal actinodromous, primary veins 5 , palmatinerved; secondary veins diverging from the lower third of the blade; tertiary veins reticulate. Staminate inflorescences thyrsiform, axillary. Pistillate inflorescences racemose, axillary. Staminate bracts $0.3-0.5 \times 0.4-0.5 \mathrm{~cm}$, lanceolate or elliptic, pubescent or tomentose. Pistillate bracts $0.3-0.8 \times 0.5-0.9 \mathrm{~cm}$, reniform or suborbicular, glabrous or pubescent. Staminate flowers with cream perianth; sepals $4,0.7-1 \times 0.4-1 \mathrm{~mm}$, elliptic or obovate, glabrous to sparsely strigose; corolla $0.3-0.9 \times 0.5-0.8$ $\mathrm{mm}$, patelliform or cupuliform, glabrous; stamens connate, forming a claviform synandrium $(0.2-0.4 \mathrm{~mm}$ long), anthers 4-6. Pistillate flowers with greenish perianth; sepal $1.4-1.6 \times 0.7-0.8 \mathrm{~mm}$, obovate, dorsally pubescent; petal $0.5-0.9 \times 0.6-1 \mathrm{~mm}$, deltoid or suborbicular, glabrous; ovary $0.6-0.9 \times 0.4-0.7 \mathrm{~mm}$, gibbous-turbinate, pubescent; stigma deltoid. Drupes $0.4-0.8 \times 0.4-0.7 \mathrm{~cm}$, obovoid, red, pubescent; endocarp with lateral and dorsoventral, tuberculate projections.

Examined material: BRAZIL, PARÁ, Barcarena, 05.II.2017, fl., J. S. Sousa 736 (MG); Canaã dos Carajás, road that connects S11D to Serra Norte, 2.XII.2015, fl., K. C.J. Rocha et al. 59 (MG).

Additional examined material: BRAZIL, AMAZONAS, Camatian, 24.I.1949, fr., R.L. Fróes 23992 (IAN, NY); São Paulo de Olivença, basin of creek Belém, 26.X.1936, fl., B. A. Krukoff 9038 (NY); São Paulo de Olivença, IV.1945, fl., R. L. Fróes 20737 (IAN); Tabatinga, 30.XI.1945, fl., J. M. Pires \& G. A. Black 939 (IAN, NY); Tabatinga, 30.XI.1945, fl., J.M. Pires \& G. A. Black 947 (IAN, NY); Tabatinga, 30.XI.1945, fr., J. M. Pires \& G. A. Black 949 (NY); Tefé, 07.XI.1948, fl., J. M. Pires 1377 (IAN, NY).

Distribution: Brazil, Colombia, Ecuador, Paraguay, Peru and Uruguay (MBG 2019, Rhodes 1975). In Brazil, this species occurs in Acre, Amazonas, Pará, Rondônia and Goiás, in the Amazonia and Cerrado phytogeographic domains (BFG 2015, Lima \& Teixeira 2018).

Among the Cissampelos species in Pará, C. laxiflora is mainly distinguished by its thyrsiform staminate inflorescence and gibbous-turbinate ovary.

Cissampelos ovalifolia DC., Syst. Nat. 1:537. 1817. (Fig. 2 A-C)

Xylopodial herb, 27-90 cm tall; stem erect, velutinous; rhytidome greenish, not fissured. Petiole 0.4-1.2 × 1-2 mm, cylindrical, not canaliculate, basifixed, velutinous; pulvinus twisted only in the distal region. Leaf blade 2.4-9.8 $\times$ $2.2-7.3 \mathrm{~cm}$, basifixed, oval or suborbicular, subcoriaceous, flat, adaxial surface pubescent or velutinous, abaxial surface velutinous; apex mucronate, obtuse or retuse; base entire, obtuse; margin entire, repand, not revolute, velutinous. Venation basal actinodromous, primary veins 5, palmatinerved; secondary veins diverging from the lower third of the blade; tertiary veins reticulate. Staminate inflorescences paniculiform, axillary or terminal. Pistillate inflorescences in simple racemes, axillary. Staminate bracts $0.5-1.5 \times 0.2-0.5 \mathrm{~cm}$, elliptic, velutinous. Pistillate bracts $0.3-1.6 \times 0.3-1.7 \mathrm{~cm}$, oval, reniform or cordiform, velutinous. Staminate flowers with greenish-yellow perianth; sepals $4-5,0.9-2 \times 0.7-1.6 \mathrm{~mm}$, elliptic or obovate, sparsely sericeous; corolla $0.5-1.8 \times 0.8-1.8$ $\mathrm{mm}$, ciatiform or patelliform, papillose; stamens connate, forming a claviform synandrium $(0.2-0.6 \mathrm{~mm}$ long), anthers 4. Pistillate flowers with greenish-yellow perianth; sepal 0.9-2 × 0.7-1.6 mm, elliptic or trullate, sericeous; petal $0.7-1.8 \times 0.9-1.8 \mathrm{~mm}$, flabelliform or reniform, glabrous; ovary $0.4-1.3 \times 0.4-0.9 \mathrm{~mm}$, gibbous, velutinous; stigma narrow-triangular. Drupes $0.6-0.9 \times 0.4-0.8 \mathrm{~cm}$, obovoid, orangish to red, pubescent; endocarp with slightly denticulate, dorsal and lateral projections.

Examined material: BRAZIL, PARÁ, Alenquer, Cerrado vegetation, 07.I.2009, fl., L .C. B. Lobato et al. 3717 (MG); Altamira, Rio Fresco, 27.VII.1962, N. T. Silva 661 (IAN); 

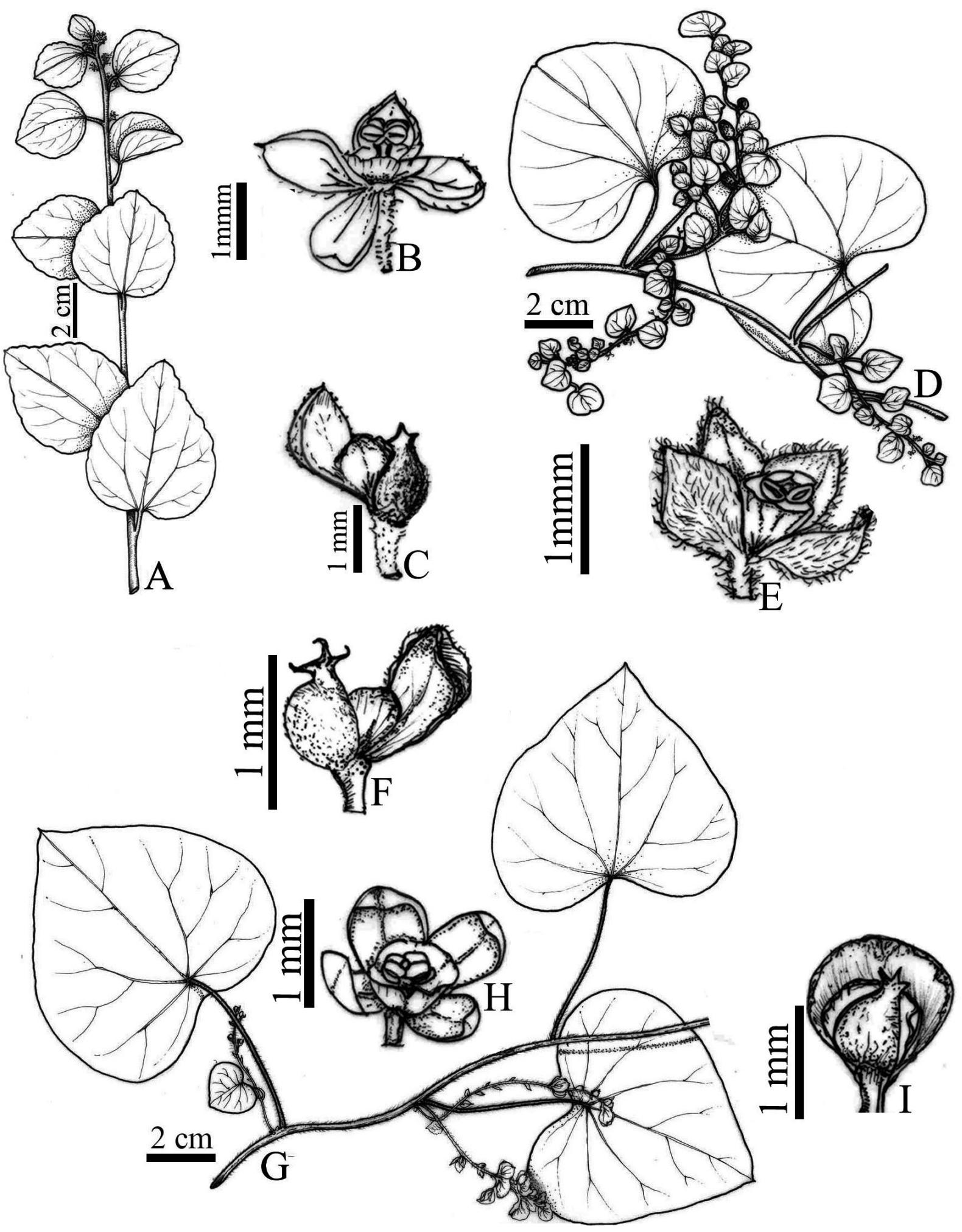

Figura 2. Species of Cissampelos in Pará state, Brazil. A-C. Cissampelos ovalifolia: A. branch with staminate flowers; B. staminate flower; C. pistillate flower. D-F. Cissampelos parreira: D. branch with pistillate inflorescences; E. staminate flower; F. pistillate flower. G-I. Cissampelos tropaeolifolia: G. branch with pistillate inflorescences; H. staminate flower; I. pistillate flower. 
Moju, 23.III.2017, fl., J. S. Sousa 745 (MG); Monte Alegre, 15.XII.1908, fl., A. Ducke 9973 (MG); Monte Alegre, Serra do Ereré, 15.V.1953, D. A. Lima 53-1596 (IAN); Óbidos, firm field of Cicandatuba, São José farm, 26.VII.1912, fl., A. Ducke 12060 (MG); Santarém, 30.I.1945, fl., R. L. Fróes 20458 (IAN); Santarém, X.2012, fl., F. C. A. Lucas 263 (MFS); Santarém, Alter do Chão, Serra do Giz, 11.III.1909, fl. \& fr., A. Ducke 10322 (MG); Santarém, Alter do Chão, 15.XII.1978, fl. \& fr., U. N. Maciel \& M.R. Cordeiro 369 (MG); Serra do Cachimbo, 17.XII.1956, fl., J. M. Pires et al. 6431 (IAN).

Distribution: Bolivia, Brazil, Colombia, Guyana, Paraguay, Suriname and Venezuela (MBG 2019, Rhodes 1975). In Brazil, this species occurs in Amazonas, Pará, Roraima, Tocantins, Bahia, Ceará, Maranhão, Paraíba, Piauí, the Distrito Federal, Goiás, Mato Grosso do Sul, Mato Grosso, Minas Gerais, São Paulo, Paraná and Santa Catarina, in the Amazonia, Caatinga, Cerrado, Atlantic Forest and Pantanal phytogeographic domains (BFG 2015).

Even when sterile, Cissampelos ovalifolia can be easily identified because it is the only xylopodial, herbaceous species of Cissampelos. In addition to this vegetative feature, the species is also distinguished by its velutinous petiole and abaxial leaf blade surface. According to Rhodes (1975), the staminate flowers have four sepals. However, in the analyzed samples, 3-4 sepals were found, which corroborates analyses made by Costa \& Sano (2007) and Teixeira \& Amorim (2012).

\section{Cissampelos pareira L., Sp. Pl. 2:1031. 1753.} (Fig. 2 D-F)

Liana, 3.5-6 $\mathrm{m}$ tall; stem voluble, puberulent; rhytidome brown, fissured. Petiole 1.4-4.6 $\times 1-2 \mathrm{~mm}$, cylindrical, not canaliculate, basifixed, tomentose; pulvinus erect in the distal and proximal regions. Leaf blade 4-8.4 × 4.6-8.5 $\mathrm{cm}$, basifixed, cordiform or suborbicular, membranous, flat, adaxial surface strigose or sericeous, abaxial surface sericeous or tomentose; apex mucronate or retuse; base bilobed, cordate; margin rectilinear, not revolute, pubescent. Venation basal actinodromous, primary veins 5-9, palmatinerved; secondary veins diverging from the lower third of the blade; tertiary veins reticulate. Staminate inflorescences paniculiform, axillary. Pistillate inflorescences in simple racemes, axillary. Staminate bracts $0.2-1.6 \times 0.15-1.1 \mathrm{~cm}$, deltoid or subcordiform, sericeous or tomentose. Pistillate bracts $0.2-0.8 \times 0.3-1 \mathrm{~cm}$, cordiform or reniform, velutinous. Staminate flowers with greenishyellow perianth; sepals 4, 0.6-1.7 × 0.4-1.2 mm, elliptic or obovate, sericeous; corolla $0.7-1.2 \times 0.6-1.2 \mathrm{~mm}$, patelliform or cupuliform, puberulent; stamens connate, forming a claviform synandrium (0.1-0.7 $\mathrm{mm}$ long), anthers 4-6. Pistillate flowers with greenish-yellow perianth; sepal $0.7-1.8 \times 0.4-1.2 \mathrm{~mm}$, flabelliform or trullate, sericeous; petal $0.4-1.2 \times 0.4-1.5 \mathrm{~mm}$, flabelliform or reniform, sericeous; ovary $0.5-1 \times 0.4-1 \mathrm{~mm}$, globose, tomentose; stigma falciform. Drupes $0.3-0.7 \times 0.3-0.5 \mathrm{~cm}$, obovoid, red, pubescent; endocarp with lateral and dorsoventral, tuberculate projections.

Examined material: BRAZIL, PARÁ, Altamira, left bank of the Rio Iriri, 19.VIII.1986, fl., S. A. M. Souza et al. 13 (MG); Aveiro, Fordlândia, 31.X.1962, fl., E. Oliveira 2357 (IAN); Belém, adjacency of the Instituto Agronômico do Norte, 17.XII.1944, fl., R.L. Fróes \& G. A. Black 24664 (IAN).

Additional examined material: BRAZIL, MINAS GERAIS, Province of Minas Gerais, 12.II.1905, fl., J. E. B. Warming (NY); Serra do Espinhaço, ca. $12 \mathrm{~km}$ of Diamantina, road to Mendanha, 28.I.1969, fl., H. S. Irwin et al. 22756 (NY); 11.I.1905, fl., P. Clausen s.n. (NY); PARANÁ, Curitiba, Rio Atuba, 30.I.1974, fl. \& fr., R. Kummrow 213 (NY).

Distribution: The species is widely distributed from Mexico to Argentina (Costa \& Sano 2007) and also occurs in Asia and Africa (Rhodes 1975, Teixeira \& Amorim 2012). In Brazil, it occurs in Acre, Amazonas, Pará, Rondônia, Roraima, Tocantins, Bahia, Maranhão, Pernambuco, Piauí, the Distrito Federal, Goiás, Mato Grosso do Sul, Mato Grosso, Minas Gerais, São Paulo, Paraná, Rio Grande do Sul and Santa Catarina, in the Amazonia, Caatinga, Cerrado, Atlantic Forest, Pampa and Pantanal phytogeographic domains (BFG 2015).

Cissampelos pareira is well defined and easily distinguished from congeners by the tomentose petiole, erect pulvinus in the distal and proximal regions, synandrium with up to 6 anthers, globose ovary and falciform stigma.

Cissampelos tropaeolifolia DC., Syst. Nat. 1:532. 1817. (Fig. 2 G-I)

Liana, 1.5-10 m tall; stem voluble, sericeous or hirsute; rhytidome brown, striate. Petiole $4.2-5.7 \times 0.75-1 \mathrm{~mm}$, subcylindrical, canaliculate, peltate (inserted 6-16 mm from the base), sericeous or hirsute; pulvinus twisted only in the proximal region. Leaf blade 5.6-7.8 $\times 5.3-8.1 \mathrm{~cm}$, peltate, cordiform or oval, membranous, flat, adaxial surface sericeous or pubescent, abaxial surface sericeous; apex acute or mucronate; base entire or bilobed, cordate, rounded or truncate; margin crenulate, not revolute, sericeous. Venation suprabasal actinodromous, primary veins $7-12$, plinerved; secondary veins diverging from the lower third of the blade; tertiary veins reticulate. Staminate inflorescences racemose or paniculiform, axillary. Pistillate inflorescences racemose, axillary. Staminate bracts $0.3-1.5 \times 0.2-2 \mathrm{~cm}$, elliptic, sericeous. Pistillate bracts $0.3-1.5 \times 0.4-1.9 \mathrm{~cm}$, cordiform or reniform, puberulent only on the margins. Staminate flowers with white or cream perianth; sepals $4,0.8-2 \times 0.4-1.3 \mathrm{~mm}$, spatulate or obovate, glabrous; 
corolla $0.8-1.2 \times 0.9-1.5 \mathrm{~mm}$, cupuliform, glabrous; stamens connate, forming a claviform or terete synandrium (0.1-0.6 mm long), anthers 4. Pistillate flowers with greenish to yellowish perianth; sepal $0.5-2.5 \times 0.5-1.5 \mathrm{~mm}$, cucullate or suborbicular, sericeous; petal 0.3-1 $\times 0.3-0.9$ $\mathrm{mm}$, obovate or suborbicular, glabrous; ovary $0.5-0.8 \times$ 0.4-0.6 mm, gibbous, sparsely strigose; stigma narrowtriangular. Drupes $0.5-0.8 \times 0.4-0.7 \mathrm{~cm}$, obovoid, red, sparsely sericeous; endocarp with lateral and dorsoventral, tuberculate projections.

Examined material: BRAZIL, PARÁ, Conceição do Araguaia, west of Redenção, near São João stream, 12.II.1980, fl. \& Fr., T. Plowman et al. 8755 (IAN, MG, NY); Faro, floodplain of Araucaru, 21.I.1920, fl., A. Ducke 5354 (RB); Oriximiná, left bank of the Rio Jamundá, 18.I.1968, fr., M. Silva 1209 (MG).

Additional examined material: BRAZIL, ACRE, Brasiléia, Basin of Rio Acre, tributary of Rio Purus, 22.X.1977, fl., D. C. Daly 9703 (NY); Sena Madureira, Basin of Rio Purus, Nova Olinda farm, 21.X.1993, fr., M. Silveira et al. 562 (INPA, NY); Xapuri, Chico Mendes Extractive Reserve, 07.XII.1993, fl., C. Figueiredo 173 (NY); Xapuri, Chico Mendes Extractive Reserve, Seringal Pimentiera road, 14.I.2002, fl. \& fr., M. Alves et al. $2372(\mathrm{NY})$.

Distribution: Mexico, Costa Rica, Guatemala, Honduras, Nicaragua, Panama, El Salvador, Belize, Bolivia, Brazil, Colombia, Ecuador, Peru and Venezuela (MBG 2019, Rhodes 1975, Teixeira \& Amorim 2012). In Brazil, this species occurs in Acre, Amazonas, Bahia, Maranhão, Pernambuco, Goiás, Mato Grosso do Sul, Minas Gerais, Rio de Janeiro, Paraná and Rio Grande do Sul, in the Amazonia, Caatinga, Cerrado, Atlantic Forest, Pampa and Pantanal phytogeographic domains (BFG 2015, Teixeira $\&$ Amorim 2012). The species is here reported for the first time for the state of Pará.

Cissampelos tropaeolifolia can be vegetatively recognized by its leaves with a crenulate margin and sericeous abaxial surface. During its reproductive phase, this species is easily distinguished by its pistillate flowers with a cucullate or suborbicular sepal and sparsely strigose ovary and sericeous drupes.

\section{ACKNOWLEDGMENTS}

The authors thank the Museu Paraense Emílio Goeldi (MPEG) for the logistical support during the study and the Conselho Nacional de Desenvolvimento Científico e Tecnológico $(\mathrm{CNPq})$ for the scholarship.

\section{REFERENCES}

Barroso, G.M., Morim, M.P., Peixoto, A.L. \& Ichaso, C.L.F. 1999. Frutos e sementes. Morfologia aplicada à sistemática de dicotiledôneas. Universidade Federal de Viçosa, Viçosa. 443 p.

BFG. 2015. Growing knowledge: an overview of Seed Plant diversity in Brazil. Rodriguésia 66 (4): 1085-1113.

Costa, F.N. \& Sano, P.T. 2007. Menispermaceae. In Flora Fanerogâmica do Estado de São Paulo (T.S. Melhem, M.G.L. Wanderley, S.E. Martins, S.L. Jung-Mendaçolli, G.J. Shepherd \& M. Kirizawa, eds.). Instituto de Botânica, São Paulo, p: 227-236.

Gonçalvez, E.G. \& Lorenzi, H. 2011. Morfologia Vegetal. Organografia e dicionário ilustrado de morfologia das plantas vasculares. Instituto Plantarum de Estudos da Flora, São Paulo. 512 p.

Hickey, L.J. 1973. Classification of the arquitecture of dicotyledonous leaves. American Journal of Botany 60: 17-33.

Lima, C.T. \& Teixeira, M.D.R. 2018. Flora das cangas da Serra dos Carajás, Pará, Brasil: Menispermaceae. Rodriguésia 69: 147-151.

Ortiz, R.S. \& Nee, M.H. 2014. A new species of Cissampelos (Menispermaceae) from Bolivia and Paraguay. Phytokeys 38: 89-99.

Ortiz, R.D.C., Wang, W., Jacques, F.M.B. \& Chen, Z. 2016. Phylogeny and a revised tribal classification of Menispermaceae (moonseed family) based on molecular and morphological data. Taxon 65: 1288-1312.

Peña-Chocarro, M. \& Egea, J. De. 2015. Nuevos registros del género Cissampelos (Menispermaceae) para la flora Argentina. Bonplandia 24: $37-42$.

Missouri Botanical Garden (MBG). 2019. TROPICOS database. Disponível em: http://www.tropicos.org. Acessado em 27.12.2019.

Radford, A.E., Dickison, W.C. \& Massey, J.R. 1974. Vascular plant systematics. Harper \& Row, New York. 891 p.

Rizzini, C.T. 1977. Sistematização terminológica da folha. Rodriguésia 29: $103-125$.

Rhodes, D.G. 1975. A revision of the genus Cissampelos. Phytologia 30: 415-484.

Sothers, C.A., Brito, J.M., Ortiz-Gentry, R. \& Ott, C. 1999. Menispermaceae. In Flora da Reserva Ducke: Guia de identificação das plantas vasculares de uma floresta de terra-firme na Amazônia central (J.E.L.S. Ribeiro et al., eds.). INPA, Manaus, p. 190-193.

SpeciesLink. 2019. Simplesearch. Disponível em: http://www.splink. org.br/index. Acessado em 27.12.2019.

Teixeira, M.D.R. \& Amorim, A.M.A. 2012. Flora da Bahia: Menispermaceae. Sitientibus. Série Ciências Biológicas 12: 207-243.

Thiers, B. (Continuously updated). 2019. Index Herbariorum: A global directory of herbaria and associated staff. New York Botanical Garden's Virtual Herbarium. Disponível em: http:// sweetgum.nybg. org/ih/. Acessado em 27.12.2019.

Weberling, F. 1992. Morphology of flowers and inflorescences. University Press, Cambridge. 405 p. 